Frederik Bachhuber, Joerg von Appen, Richard Dronskowski, Peer Schmidt, Tom Nilges, Arno Pfitzner and Richard Weihrich*

\title{
Van der Waals interactions in selected allotropes of phosphorus
}

\begin{abstract}
Selected allotropes of phosphorus are investigated by different levels of density functional theory (DFT) calculations to evaluate the relative stability orders with a special focus on the role of van der Waals interactions. Phosphorus is an excellent reference system with a large number of allotropes. Starting from low-dimensional molecular (OD, white P) and polymer structures (1D, P nanorods) to layered (2D, black $\mathrm{P})$ and tubular structures (2D and 3D, crystalline forms of red P), covalent structure motifs are interconnected by van der Waals interactions. They are a key factor for the correct energetic description of all $\mathrm{P}$ allotropes. A comparative study is carried out within the local density approximation (LDA) and the generalized gradient approximation (GGA), with and without implementation of a dispersion correction by Grimme (GGA-D2). Our intention is to achieve a reasonable agreement of our calculations with experimental data, the plausibility of energy values, and the treatment of long-range interactions. The effect of van der Waals interactions is exemplified for the interlayer distances of black phosphorous and its electronic structure.
\end{abstract}

Keywords: allotropes; band structure; DFT; phosphorous; stability; van der Waals.

DOI 10.1515/zkri-2014-1800

Received September 1, 2014; accepted October 19, 2014; published online December 4, 2014

\footnotetext{
*Corresponding author: Richard Weihrich, Institut für Anorganische Chemie, Universität Regensburg, Universitätsstr. 31, 93040 Regensburg, Germany, E-mail: richard.weihrich@ur.de Frederik Bachhuber and Arno Pfitzner: Institut für Anorganische Chemie, Universität Regensburg, Universitätsstr. 31, 93040 Regensburg, Germany

Joerg von Appen and Richard Dronskowski: Institut für Anorganische Chemie, RWTH Aachen University, Landoltweg 1, D-52056 Aachen, Germany

Peer Schmidt: Fakultät für Naturwissenschaften, BTU CottbusSenftenberg, Großenhainer Straße 57, 01968 Senftenberg, Germany Tom Nilges: Department of Chemistry, TU München, Lichtenbergstr. 4, 85747 Garching, Germany
}

\section{Introduction}

Within the priority project SPP1415, we performed combined experimental and theoretical investigations on metastable compounds with main-group $\mathrm{V}$ elements. One part of the project dealt with the relative stabilities of polymorphs of dipnictides $\mathrm{NiPn} n_{2}(P n=\mathrm{N}, \mathrm{P}, \mathrm{As}, \mathrm{Sb}, \mathrm{Bi})$ [1] and related ternary ordering variants $M P n S(M=\mathrm{Fe}$, $\mathrm{Co}, \mathrm{Ni} ; \mathrm{Pn}=\mathrm{As}, \mathrm{Sb}$ ) [2]. The structures of the pnictogen elements themselves were studied in another part of the project with a focus on the solid solution $\mathrm{As}_{\mathrm{x}} \mathrm{P}_{1-\mathrm{x}}[3]$ and the various allotropes of phosphorus [4]. Subsequently, some of the well-known and new phosphorus allotropes have been thoroughly investigated concerning the role of van der Waals interactions for their solid structures and relative stabilities.

The element phosphorus fascinates by the occurrence of a large number of allotropic modifications in the form of layers in black $\mathrm{P}[5,6]$, tetrahedra in white $\mathrm{P}(\alpha-, \beta$-, and $\left.\gamma-P_{4}\right)[7-9]$, or tubular units in red P. Some of the red phases are still structurally unknown. Lately, pair-wise tubes were discovered and obtained in crystalline form with parallel orientation (Ruck's fibrous P [10]) - in contrast to a perpendicular arrangement in Hittorf's violet $P$ [11]. The discovery of different kinds of $P$ nanorods [12-15] extended the variety of $\mathrm{P}$ structures but left some open questions concerning their crystal structures and their place in the stability order of the P allotropes.

Phosphorus has never lost its attraction to scientist in various disciplines from nuclear medicine to inorganic chemistry [16]. Current reports include the catalytic activity of red P [17], black P as photo-detector [18], or the highly topical discovery of single-layer phosphorenes $[19,20]$. Especially in the field of solid-state chemistry, finding novel ways of preparation [21, 22] and exploration of existing and new allotropes is still a challenging task. This applies for experimentalists $[9,10,15]$ as much as for theoreticians [23-28]. For the latter, the phosphorus allotropes provide an ideal basis for the evaluation of their methods with the multifaceted appearance of the element. Despite its vast structural variety and the availability of experimental data, little attention has been given 
to the allotropes of phosphorus in terms of theoretical investigations (in contrast to the more closely examined allotropy of carbon, primarily in the forms of graphite, carbon nanotubes and diamond). Previous studies on phosphorus were mainly devoted to different approaches for the accurate modeling of, for instance, $\mathrm{P}_{2}, \mathrm{P}_{4}$, or black phosphorus [29]. Also, the treatment of van der Waals interactions was found decisive for the correct prediction of cell volumes. Nonetheless, no comparison of all P allotropes at one consistent level of theory is available up to date, nor any information on solid structures of the novel $\mathrm{P}$ nanorods. This is the reason for the present study.

As a first step, we studied the stability order of the boundary phases orthorhombic black phosphorus and trigonal grey arsenic in the solid solution $\mathrm{P}_{1-\mathrm{x}} \mathrm{As}_{\mathrm{x}}$ in a combined experimental and DFT investigation [3]. The respective stability of orthorhombic vs. high-pressure trigonal black P and vice versa of trigonal gray vs. orthorhombic black As was correctly described by GGA calculations. Surprisingly, this could not be reproduced by LDA functionals. Furthermore, the energetic order between orthorhombic black P, Hittorf's violet P and Ruck's fibrous $P$ caused confusion from experimental and calculated data. From in situ gas-phase reaction measurements, orthorhombic black $\mathrm{P}$ emerged as more stable than violet $\mathrm{P}$, but fibrous $\mathrm{P}$ more stable than black P. At this point, it becomes important to take a look at the types of interaction occurring in the various allotropes, and to analyze how they are covered by the different theoretical methods. The phosphorus allotropes serve as a benchmark case to study the performance of standard levels of DFT on lowdimensional systems with strong covalent networks that are interconnected by van der Waals forces. The latter turned out to be evident for the correct description of the entire stability range, solid structures, and the prediction of the crystal structures of the recently discovered $\mathrm{P}$ nanorod allotropes [4]. The results are evaluated in more detail herein with supplementary material and a focus on the applied methods and the treatment of dispersive interactions.

\section{Methods}

Among the established classes of $a b$ initio electronic structure methods, density functional theory (DFT) [30, 31] emerged as an efficient option for the calculation of systems with periodic unit cells. In its early stages, DFT had to deal with two major drawbacks: the deliberate search for improved exchange-correlation functionals (although the performance of the LDA was surprisingly good for many different systems) and the poor description of long-range van der Waals or dispersion interactions. Concerning the development of new or improved exchange-correlation functionals, numerous innovations have been made. The establishment of the GGA in combination with the projector augmented wave method (PAW) ranges among the most promising advances. Astonishing for layered structures with strong van der Waals interactions such as graphite, calculations performed with the LDA still yielded better results than with the GGA functional [32]. A comparative study by Girifalco and Hodak [33] concluded that utilizing the LDA functional in fact takes account of interactions between the graphene layers but not on the basis of van der Waals forces. Further limitations of common time-independent DFT methods applying both LDA and GGA functionals to correctly describe graphite became apparent [34-36] with considerable disagreement between calculated and experimental data.

As a consequence, strong efforts have been made to tackle the issue of a proper description of dispersion interactions. This led to the development of modified density functionals claiming to account for both short- and long-range interactions, such as the nonlocal correlation functional (NLCF) [36-39]. A powerful alternative is the adiabatic connection fluctuation dissipation theorem within the random-phase approximation (RPA) [40-42]. According to a review on the calculation of dispersion energies by Dobson and Gould [43] and a comparative study of the performance of different many-body perturbation and advanced density-functional theory techniques on selected layered structures by Björkman et al. [44], RPA methods deliver most reliable results but at very large computational costs.

Involving the generally well-established functionals LDA and GGA, atom-pairwise dispersion corrections as suggested by Grimme [45] (DFT-D) turned out to be very (cost-)effective at an adequate accuracy. This was in particular shown for the combination with the GGA approach according to Perdew-Burke-Ernzerhof (PBE) for graphitelike and other layered structures [46, 47]. More precisely, there is an enhanced agreement of calculated and experimental cell geometries even for systems where dispersion interactions play a minor role such as diamond or sodium chloride. Energetically, such enhanced DFT calculations also allow to correctly model various three-dimensional $\mathrm{TeO}_{2}$ polymorphs which include dispersion interactions and which are close to each other in Gibbs free energy by $<1 \mathrm{~kJ} / \mathrm{mol}$ [48].

A recent study on white phosphorus [49] confirmed the application of the corrections by Grimme in combination with the GGA/PBE (GGA-D2) to yield more accurate results compared to the common use of the GGA or the LDA. Furthermore, the functionals were evaluated for black phosphorus in terms of electronic structure calculations [50, 51]. Dispersion-corrected atom-centered potentials (DCACPs), which are well-suited for the combination with the GGA/PBE, were also reported to improve the calculations for black and white phosphorus [52]. The effect of the common functionals with and without dispersion correction on the residual phosphorus allotropes remains to be analyzed. Herein, we examine the performance of the cost-effective, versatile, and widely used functionals LDA and GGA - with a focus on the dispersion-corrected routine by Grimme (GGA-D2) - in terms of cell parameters and relative stabilities of the phosphorus allotropes.

\section{Computational details}

The first-principles electronic structure calculations were carried out within the framework of DFT with exchangecorrelation functionals in the generalized gradient 
approximation (GGA) [53, 54] according to Perdew-BurkeEnzerhof (PBE) [55] and in the local density approximation according to Perdew and Zunger (LDA) [56]. Crystal structures and energies were additionally calculated by means of long-range dispersion correction by Grimme (DFT-D2) $[45,57]$. Full geometry optimizations were executed with the Vienna Ab initio Simulation Package (VASP) [58-61], atomic-site and cell parameters were therefore allowed to fully relax with the conjugate gradient algorithm. The interactions between ions and electrons were described by the projector-augmented-wave (PAW) method $[62,63]$ with a cutoff energy of $500 \mathrm{eV}$. All calculations were performed in several subsequent steps with increasing $k$-grid meshes (depending on the size of the unit cell) between $2 \times 2 \times 2$ and $12 \times 12 \times 12$ to reach sufficient accuracy. Particularly for large unit cells, this offers an additional verification of the performed calculations. A structure optimization was considered to be converged with a difference in total energy of $<1 \times 10^{-6} \mathrm{eV}$ and a maximum Hellmann-Feynman force of $1 \times 10^{-4} \mathrm{eV} / \mathrm{A}$. The final values of the total energies were obtained with energy differences (between last and second to last step) of $<1 \times 10^{-3} \mathrm{eV}$ per formula unit.

\section{Crystal structures}

Representative sections of the crystal structures of phosphorus allotropes along with characteristic structural fragments are shown in Figure 1, and detailed structural data are given in Table 1 in the following section. The thermodynamically stable allotrope at room temperature is orthorhombic black phosphorus [64]. It is composed of puckered layers built of $\mathrm{P}_{6}$ rings in chair conformation (with endo connection of the rings). The trigonal highpressure modification of phosphorus is related to black phosphorus and exhibits the crystal structure of gray arsenic. It also consists of $\mathrm{P}_{6}$ rings in chair conformation but with different connection of the rings (exo). The staggered manner in which the layers are stacked results in denser packing with smaller interlayer distances. Further increase of pressure leads to another high-pressure modification with simple cubic arrangement of the phosphorus atoms.

Likewise, the primary building units of the tubular (red) allotropes are six-membered rings of phosphorus, yet in boat conformation. Instead of a two-dimensional connection in the form of layers, capped strands/ tubes are formed. The building units of fibrous and violet phosphorus are equivalent but interconnected in different ways. In fibrous phosphorus, always two of the units are combined to parallel double tubes. A crosswise connection of the tubes leads to Hittorf's violet phosphorus. Three kinds of isolated strands with more or less distinct distortion could be synthesized in CuI matrices, namely $(\mathrm{CuI})_{8} \mathrm{P}_{12},(\mathrm{CuI})_{3} \mathrm{P}_{12}$, and $(\mathrm{CuI})_{2} \mathrm{P}_{14}$. The structure of the single strands is clearly related to the tubular units in fibrous and violet $\mathrm{P}$. Instead of an interconnection, additional $\mathrm{P}$ atoms are incorporated into the tubes to maintain trivalent phosphorus on all positions. The structures of the
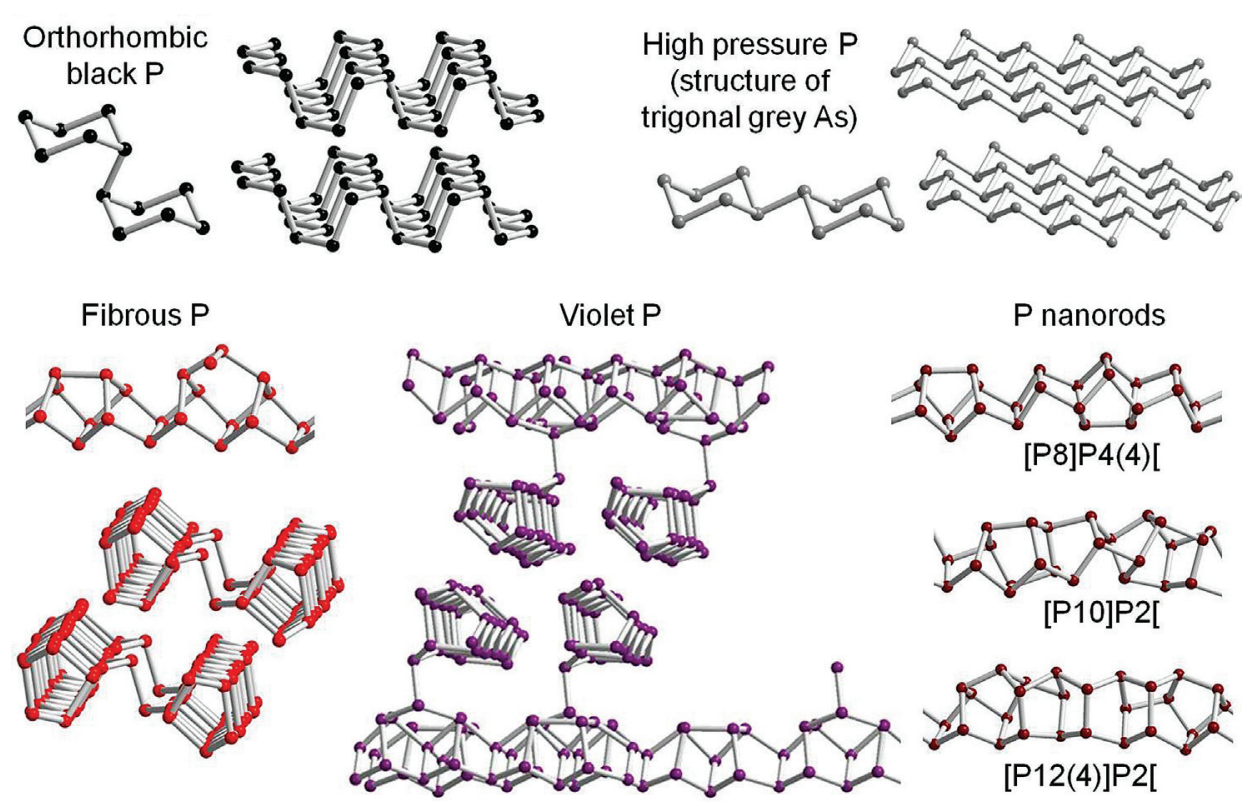

Fig. 1: Characteristic structural fragments of layered and tubular P allotropes. 
Tab. 1: LDA-, GGA-, and GGA-D2-calculated structural data and energy values of the allotropes of phosphorus obtained by full lattice optimizations.

\begin{tabular}{|c|c|c|c|c|c|c|c|c|c|c|c|c|c|c|}
\hline P-allotrope & $\begin{array}{l}\text { Space } \\
\text { group (no.) }\end{array}$ & & $a / \AA ̊$ & $b / \AA$ & $c / \AA$ & $b / a$ & $c / a$ & $c / b$ & $\alpha / /^{\circ}$ & $\beta /^{\circ}$ & $\gamma /{ }^{\circ}$ & $V / \AA^{3}$ & $V / \AA^{3} /$ atom & $\overline{E / \mathrm{kJ} / \mathrm{mol}}$ \\
\hline \multirow[t]{4}{*}{ White $(\beta)$} & $P \overline{1}(2)$ & Exp. [8] & 5.48 & 10.79 & 10.96 & 1.97 & 2.00 & 1.02 & 94.29 & 99.65 & 100.68 & 623.8 & 26.0 & \\
\hline & & LDA & 5.20 & 10.15 & 10.20 & 1.95 & 1.96 & 1.01 & 93.95 & 97.43 & 100.63 & 521.8 & 21.7 & -557.4 \\
\hline & & GGA & 5.92 & 11.63 & 11.74 & 1.96 & 1.98 & 1.01 & 94.51 & 99.42 & 100.76 & 778.9 & 32.5 & -508.1 \\
\hline & & $-\mathrm{D} 2$ & 5.41 & 10.68 & 10.88 & 1.97 & 2.01 & 1.02 & 94.25 & 99.76 & 100.99 & 604.5 & 25.2 & -519.6 \\
\hline \multirow[t]{4}{*}{ White $(\gamma)$} & $C 2 / m(12)$ & Exp. [9] & 9.17 & 8.34 & 5.43 & 0.91 & 0.59 & 0.65 & & 90.31 & & 415.5 & 26.0 & \\
\hline & & LDA & 8.53 & 7.62 & 5.22 & 0.89 & 0.61 & 0.69 & - & 94.32 & - & 338.9 & 21.2 & -557.9 \\
\hline & & GGA & 9.79 & 9.07 & 5.92 & 0.93 & 0.60 & 0.65 & - & 91.32 & - & 525.7 & 32.9 & -508.1 \\
\hline & & $-\mathrm{D} 2$ & 9.09 & 8.20 & 5.32 & 0.90 & 0.59 & 0.65 & - & 90.19 & - & 401.2 & 25.1 & -520.1 \\
\hline \multirow[t]{4}{*}{ Simple cubic (hp) } & $P m \overline{3} m(221)$ & Exp. [6] & 2.38 & & & & & & & & & 13.4 & 13.4 & \\
\hline & & LDA & 2.35 & - & - & - & - & - & - & - & - & 13.0 & 13.0 & -582.2 \\
\hline & & GGA & 2.38 & - & - & - & - & - & - & - & - & 13.4 & 13.4 & -508.4 \\
\hline & & $-\mathrm{D} 2$ & 2.37 & - & - & - & - & - & - & - & - & 13.3 & 13.3 & -533.4 \\
\hline \multirow[t]{4}{*}{ Trigonal (hp) } & $R \overline{3} m(166)$ & Exp. [6] & 3.52 & & & & & & 57.25 & & & 29.0 & 14.5 & \\
\hline & & LDA & 3.42 & - & - & - & - & - & 57.52 & - & - & 26.6 & 13.3 & -584.5 \\
\hline & & GGA & 3.58 & - & - & - & - & - & -56.53 & - & - & 29.8 & 14.9 & -513.6 \\
\hline & & $-\mathrm{D} 2$ & 3.46 & - & - & - & - & - & 57.40 & - & - & 27.6 & 13.8 & -536.8 \\
\hline \multirow[t]{4}{*}{ Black } & Cmca (64) & Exp. [5] & 3.31 & 10.48 & 4.38 & 3.16 & 1.32 & 0.42 & & & & 151.9 & 19.0 & \\
\hline & & LDA & 3.31 & 10.16 & 4.14 & 3.07 & 1.25 & 0.41 & - & - & - & 139.1 & 17.4 & -580.6 \\
\hline & & GGA & 3.31 & 11.21 & 4.55 & 3.39 & 1.38 & 0.41 & - & - & - & 168.6 & 21.1 & -518.4 \\
\hline & & $-\mathrm{D} 2$ & 3.31 & 10.48 & 4.41 & 3.17 & 1.33 & 0.42 & - & - & - & 153.8 & 19.2 & -537.5 \\
\hline \multirow[t]{4}{*}{ Violett } & $P 2 / C(13)$ & Exp. [11] & 9.21 & 9.15 & 22.60 & 0.99 & 2.45 & 2.47 & & 106.10 & & 1829.8 & 21.8 & \\
\hline & & LDA & 9.10 & 9.00 & 21.62 & 0.99 & 2.38 & 2.40 & - & 106.64 & - & 1697.3 & 20.2 & -579.0 \\
\hline & & GGA & 9.27 & 9.26 & 24.19 & 1.00 & 2.61 & 2.61 & - & 104.31 & - & 2011.8 & 24.0 & -521.3 \\
\hline & & $-\mathrm{D} 2$ & 9.23 & 9.16 & 22.59 & 0.99 & 2.45 & 2.47 & - & 106.1 & - & 1836.1 & 21.9 & -537.4 \\
\hline \multirow[t]{4}{*}{ Fibrous } & $P \overline{1}(2)$ & Exp. [10] & 11.63 & 12.20 & 7.08 & 1.05 & 0.61 & 0.58 & 71.07 & 73.69 & 84.17 & 911.3 & 21.7 & \\
\hline & & LDA & 11.38 & 11.45 & 6.77 & 1.01 & 0.59 & 0.59 & 72.59 & 76.04 & 86.65 & 816.8 & 19.4 & -578.9 \\
\hline & & GGA & 12.28 & 13.16 & 7.89 & 1.07 & 0.64 & 0.60 & 69.37 & 71.19 & 77.02 & 1121.1 & 26.7 & -521.8 \\
\hline & & $-\mathrm{D} 2$ & 11.63 & 12.17 & 7.00 & 1.05 & 0.60 & 0.58 & 71.46 & 74.02 & 84.89 & 903.0 & 21.5 & -537.3 \\
\hline \multirow[t]{3}{*}{ [P8]P4(4)[ } & $P 2_{1}(4)$ & LDA & 9.49 & 9.98 & 10.69 & 1.05 & 1.13 & 1.07 & - & 100.06 & - & 1004.9 & 20.9 & -574.9 \\
\hline & & GGA & 10.51 & 12.43 & 13.04 & 1.18 & 1.24 & 1.05 & - & 102.87 & - & 1597.3 & 33.3 & -519.6 \\
\hline & & $-\mathrm{D} 2$ & 9.47 & 10.61 & 11.25 & 1.12 & 1.19 & 1.06 & - & 99.65 & - & 1114.2 & 22.9 & -533.5 \\
\hline \multirow[t]{3}{*}{ [P10]P2[ } & $P 2_{1} / C(14)$ & LDA & 10.70 & 13.74 & 6.50 & 1.28 & 0.61 & 0.47 & - & 94.55 & - & 952.1 & 19.8 & -576.1 \\
\hline & & GGA & 12.50 & 14.11 & 7.54 & 1.13 & 0.60 & 0.53 & - & 96.16 & - & 1319.1 & 27.5 & -519.4 \\
\hline & & $-\mathrm{D} 2$ & 12.96 & 14.16 & 6.48 & 1.09 & 0.50 & 0.46 & - & 105.12 & - & 1047.0 & 21.8 & -534.3 \\
\hline \multirow[t]{3}{*}{ [P12(4)]P2[ } & $P 2_{1} / c(14)$ & LDA & 6.79 & 10.91 & 16.12 & 1.61 & 2.37 & 1.48 & - & 113.64 & - & 1093.4 & 19.5 & -574.9 \\
\hline & & GGA & 7.49 & 12.54 & 16.59 & 1.67 & 2.22 & 1.32 & - & 113.81 & - & 1380.2 & 24.6 & -519.0 \\
\hline & & $-\mathrm{D} 2$ & 7.01 & 11.43 & 16.64 & 1.63 & 2.37 & 1.46 & - & 114.44 & - & 1201.1 & 21.4 & -535.4 \\
\hline
\end{tabular}

three different CuI-free nanorod types were elucidated in [4] and described, based on their repeating units, as [P8] P4(4)[, [P10]P2, and [P12(4)]P2[according to [23]. Although parallel stacking of the nanorods could be proven, the packing ability is lowered because of the distorted linearity of the tubes. Improved packing behavior towards the other tubular and the layered allotropes is reflected by smaller densities. Amorphous red phosphorus can be seen as an intermediate phase composed of an irregular network of tubular building units.

The packing of the tetrahedra in white phosphorus involves a high degree of intermolecular (non-covalent) bonding so it exhibits the smallest density. The three modifications of white phosphorus correspond to three different ways of packing the tetrahedra. As accurate single crystal data of the plastic $\alpha$ modification of white phosphorus is not accessible, only the $\beta$ and the $\gamma$ modification are considered here.

Whether there are layers, tubes, fibers, or tetrahedra, the building units themselves are formed by strong covalent bonds but they are held together by dispersive interactions. It is of great interest how the widely used functionals LDA and GGA account for these interactions in terms of a correct reproduction of the cell geometry and relative stabilities. Just as interesting is the impact of the dispersion correction on the calculations. 


\section{Results and discussion}

To point out the changing stability order of the $P n=P$, As crystal structures, calculations were perfomed on orthorhombic and trigonal layered structures of $\mathrm{P}$ and As $[3,4]$. Similar to our earlier work on $\mathrm{TN}_{2}(T=\mathrm{C}, \mathrm{Si}, \mathrm{Ge})$ compounds [65], calculations with the LDA functional underestimate lattice parameters, which are mostly overestimated by the GGA. In case of As, both functionals predict the experimentally known trigonal structure as more stable than the orthorhombic structure of black P. In agreement with experiment, GGA also predicts orthorhombic $\mathrm{P}$ as more stable than high-pressure trigonal P. The LDA calculations, however, predict the contrary, i.e., trigonal $\mathrm{P}$ more stable than orthorhombic P. In contradiction with experiment, it must be taken as an LDA artefact which has not been fully understood to date [29].

Nonetheless, the GGA calculations also cause discrepancies, which becomes apparent in the energy-volume curves for black and violet phosphorus in Figure 2 (LDA-, GGA-, and GGA-D2-based calculations). While the energy-volume plots obtained with the LDA exhibit a distinct minimum, an accurate determination of such a minimum is hardly feasible for the GGA calculations as the curves are much flatter. The fact that the curves remain flat with increasing volumes indicates a minor role of interlayer interactions. In contrast, the energy-volume curves acquired with dispersion correction exhibit a trend similar to the ones obtained with the LDA indicating a distinct minimum. Not only do the GGA-D2-determined cell volumes agree well with the full lattice optimizations but they also match the experimental values more accurately (an underestimation is yielded with LDA). This corrective effect of GGA-D2 was also reported for graphite [46]. Looking at the energy differences between black and violet phosphorus, the application of GGA-D2 vs. GGA even leads to a change of the stability order. This fact will also be subject of the discussion below.

Finally, the dispersion energy part of the total energy for black P is explicitly determined. Similar to experiments on graphite, the interlayer distance was modified only by variation of the crystallographic $b$ axis. The observations of the $E-V$ fittings strongly correlate with the LDA-, GGA-, and GGA-D2-calculated interlayer binding energies that are denoted in Figure 3. They serve as a meaningful indicator for the treatment of van der Waals interactions with the different functionals. Accordingly, LDA takes interlayer interactions much more into account than GGA with an interlayer binding energy of almost $10 \mathrm{~kJ} / \mathrm{mol}$ vs. about $1 \mathrm{~kJ} / \mathrm{mol}$, respectively. This correlates with the
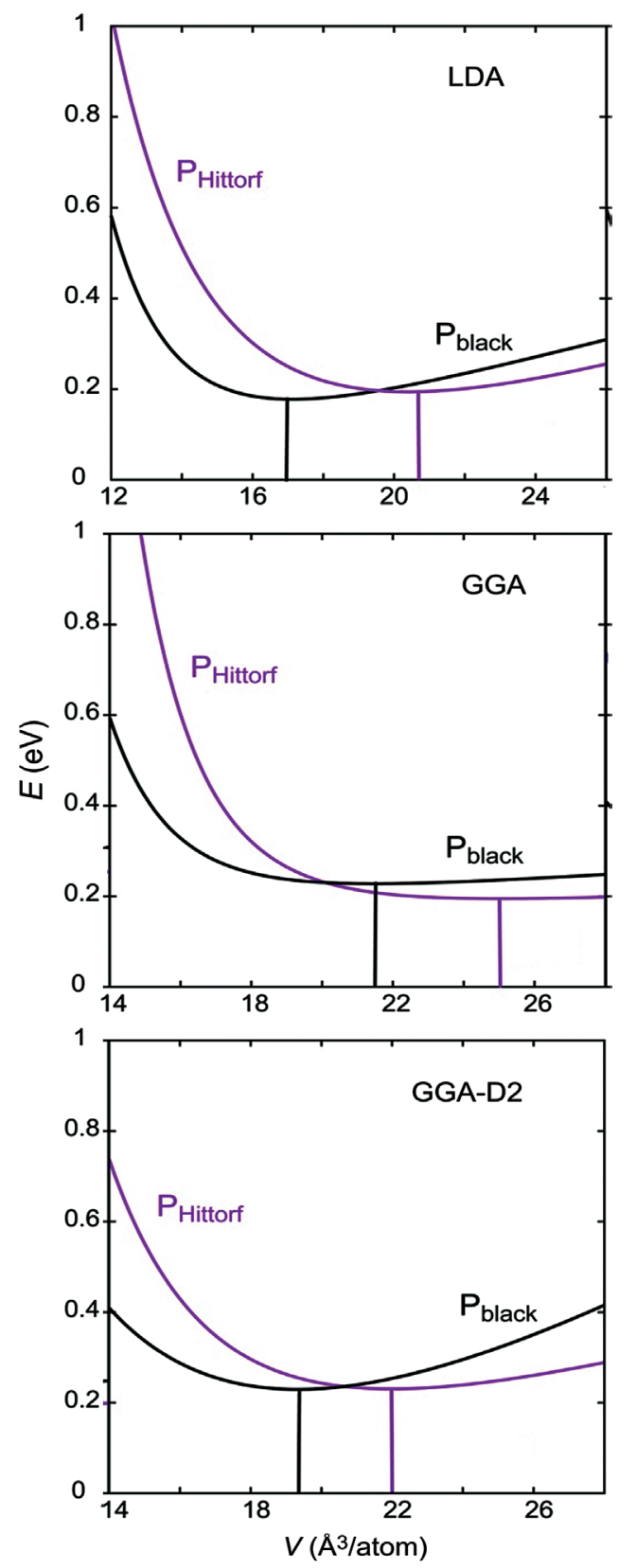

Fig. 2: LDA-, GGA-, and GGA-D2-calculated energy-volume curves for orthorhombic black $P$ and violet/Hittorf's phosphorus.

calculated interlayer distances of $2.92 \AA$ (corresponding to $3.41 \AA$ as shortest P-P distance between two layers) for LDA and $3.48 \AA$ ( $3.94 \AA$ ) for GGA that are situated $6.1 \%$ below and $11.9 \%$ above the experimental value of $3.11 \AA$ (3.59 $\AA$ ). Overbinding and underbinding are well-known effects for LDA and GGA, respectively, and they are distinctly reflected for the treatment of interlayer binding/van der Waals interactions. In contrast, the values obtained from the GGA-D2 calculations are very reasonable with an interlayer binding energy of about $7 \mathrm{~kJ} / \mathrm{mol}$ and an interlayer 


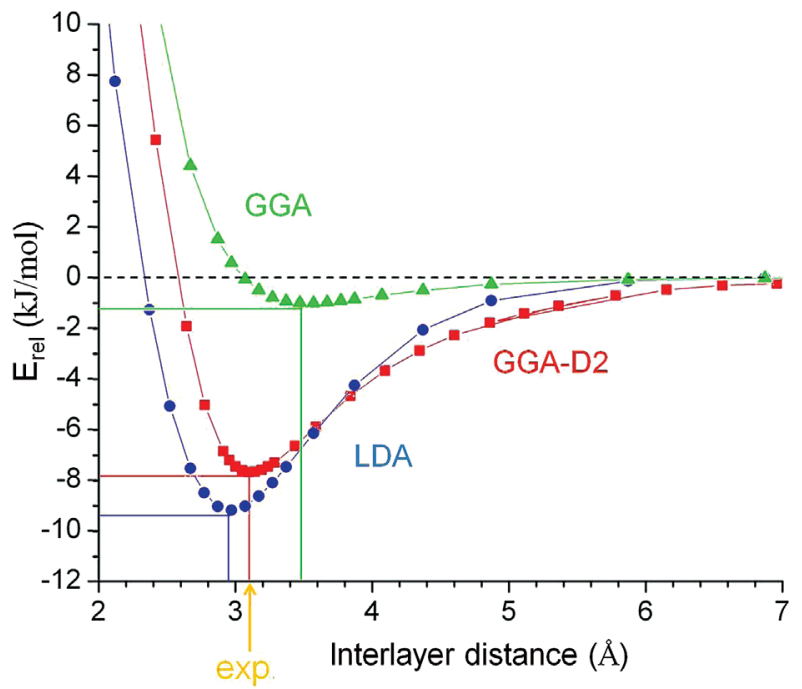

Fig. 3: Interlayer distance (corresponding to a varying $b$-axis length) versus energy plot of orthorhombic black $P$ to estimate the interlayer binding energy for LDA-, GGA-, and GGA-D2- based calculations.

distance of $3.13 \AA$ ( $3.60 \AA$ ) for GGA-D2, which is in excellent agreement with the experimental value $(+0.6 \%)$.

The results of the LDA, GGA, and GGA-D2 calculations for selected allotropes of phosphorus (except white $\mathrm{P}$, just the nanorod type [P10]P2[) are merged in an energy-volume plot in Figure 4, and the associated data are given in Table 1. It contains detailed cell parameters of full lattice optimizations alongside with a comparison

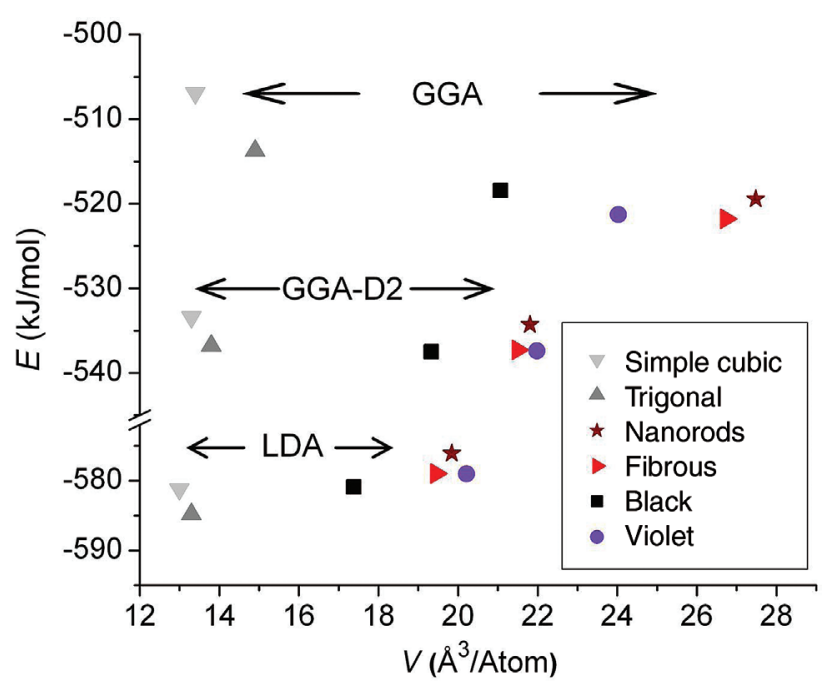

Fig. 4: LDA- (bottom), GGA- (top), and GGA-D2 (middle)-calculated energy-volume plot (total energies) of selected phosphorus allotropes. Note that just one nanorod form ([P10]P2D) is given and white phosphorus is not displayed here for clarity reasons (calculated energy and volume data are given in Table 1, along with corresponding structural data). with experimental data. As the experimental volumes of cubic and trigonal $\mathrm{P}$ were measured under pressures of $12 \mathrm{GPa}$ and $9 \mathrm{GPa}$ [6], the calculated values were adjusted to the same conditions. It is interesting to see that the simple cubic modification is the only case of agreeing GGA and GGA-D2 values due to negligible dispersive interactions.

Regarding unit cell shapes and sizes, both LDA- and GGA-based calculations demonstrate the often-quoted over- and underbinding, respectively. The underbinding of the GGA is clearly more pronounced, in particular for the tubular structures. In general, the contribution of GGA-D2 leads to a much better agreement with experimental data. The necessity of the dispersion correction becomes particularly obvious by taking the volume contraction of the tubular allotropes into account, in contrast to the pure GGA values. The volume contraction effects rise from violet $\mathrm{P}$ to fibrous $\mathrm{P}$ and the nanorods, in line with the decreasing amount of intertubular covalent connections. For violet $\mathrm{P}$, there is a relatively small contraction as the structure is already held together in interlocked grids. The volume contraction is more distinct for the nanorods and fibrous $\mathrm{P}$ as there is no prearrangement in stacking of the single and double strands caused by covalent bonds. Altogether, the cell volumes (per atom) of the tubular structures are relocated to nearly congruent values. This is very plausible if the strong relationships between the building units are kept in mind. LDA-calculated volumes are too small compared with experimental data.

Concerning the energy values, there are prominent discrepancies between the different approaches. Overall reasonable results can be found for the tubular phases. Fibrous and violet phosphorus are almost equal in energy as also reported before [10]. According to all our calculations, they are lower in energy than the nanorods. This agrees well with a comprehensive investigation [23] that predicted the building units of Hittorf's (and fibrous) phosphorus as energetically most favorable of all predicted tubular phosphorus chains. That paper also described the energetic order of the nanorod fragments with rising stability from [P8]P4(4)[to [P12(4)]P2[and [P10]P2[. This is now confirmed for the polymer structures of single rods (see Figure 5). [P8]P4(4)[emerges as more than $10 \mathrm{~kJ} / \mathrm{mol}$ higher in energy with $\mathrm{P}_{4}$ units destabilizing the covalent network. The energetic situation changes, however, for the crystal structures of the P nanorods. Due to a decreasing linearity/ regularity of the tubes from [P8]P4(4)[to [P12(4)] $\mathrm{P} 2[$ and [P10]P2[(cf. [4]), the energy gain for rod packing, i.e., the amount of van der Waals bonding interaction between the tubes, gets lower in this row, and therefore the energetic order must be modified accordingly. 


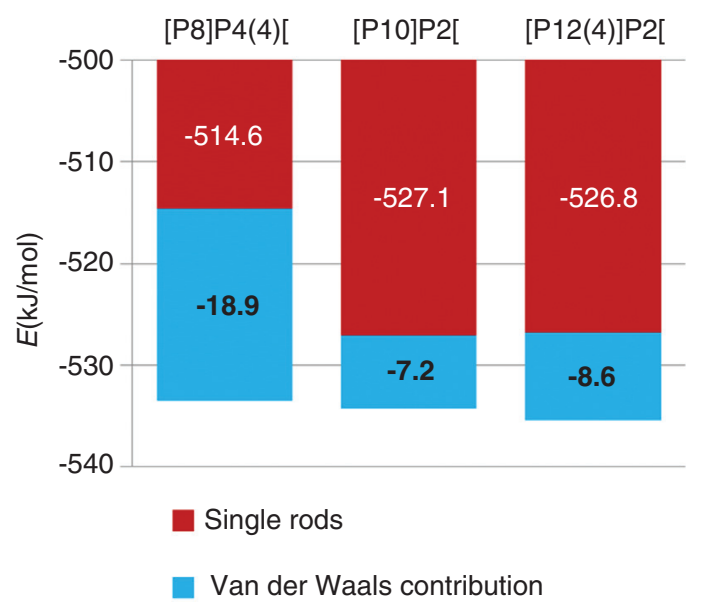

Fig. 5: Bar chart of the GGA-D2-calculated energies of single nanorods (dark red bars) derived from (Cul) ${ }_{8} \mathrm{P}_{12}$ ([P8]P4(4)[), (Cul) ${ }_{3} \mathrm{P}_{12}$ ([P10]P2[), and (Cul) ${ }_{2}$ 14 $_{14}([\mathrm{P} 12(4)] \mathrm{P} 2[)$ and the total energies of their crystal structures (total bars). The energy difference corresponds to the energy gain for packing the rods, i.e. for VdWi (bright blue bars).

While the description of the tubular structures per se is fairly consistent for the different functionals, relative energy differences between the other allotropes disagree significantly. As known from experiment, black phosphorus is the thermodynamically stable modification, and its stability increases with decreasing temperature. The GGA calculations, however, predict all tubular allotropes to be lower in energy than black P. Once again, a remedy is provided by the dispersion correction that causes a shift of the relative stabilities towards the expected order. The energy gain with GGA-D2 rises from the nanorods to fibrous and violet phosphorus in accordance with a rising amount of van der Waals interactions, even though the LDA calculations confirm the black allotrope as energetically more favorable than the tubular structures. A significant error emerges concerning the stabilities of the high-pressure forms of phosphorus. In addition to the erroneous prediction of the trigonal structure of arsenic as 'lowest-energy' phase, the simple cubic arrangement is shifted towards the energy region of black phosphorus. As a general trend (and in contrast to the GGA calculations), the LDA seems to prefer denser structures considerably. This trend is in line with previous studies on other textbook phases like carbon or BN [66].

In summary, the cell geometries and the relative energies are in best agreement with experimental values for GGA-D2. As far as absolute energy values are concerned, a comparison of black and white phosphorus with a relatively large energy difference is reasonable. The experimentally found transition enthalpy is $21.2 \pm 2.1 \mathrm{~kJ} / \mathrm{mol}$ at ambient conditions. The corresponding GGA value is just $10.3 \mathrm{~kJ} / \mathrm{mol}$ which gets shifted to $17.4 \mathrm{~kJ} / \mathrm{mol}$ for the GGA-D2 calculations. An even better agreement with the experimental value is observed for the LDA value of 23.5 $\mathrm{kJ} / \mathrm{mol}$. This is accompanied by the fact, however, that LDA calculations generally yield higher energy differences and erroneously predict the high-pressure modifications as more stable than black P.

In this regard, it is also worth having a look at the effect of the applied functional on the electronic structure using the example of black phosphorus. The band structures around the Fermi level are displayed in Figure 6. A failure of the LDA can be observed when examining the
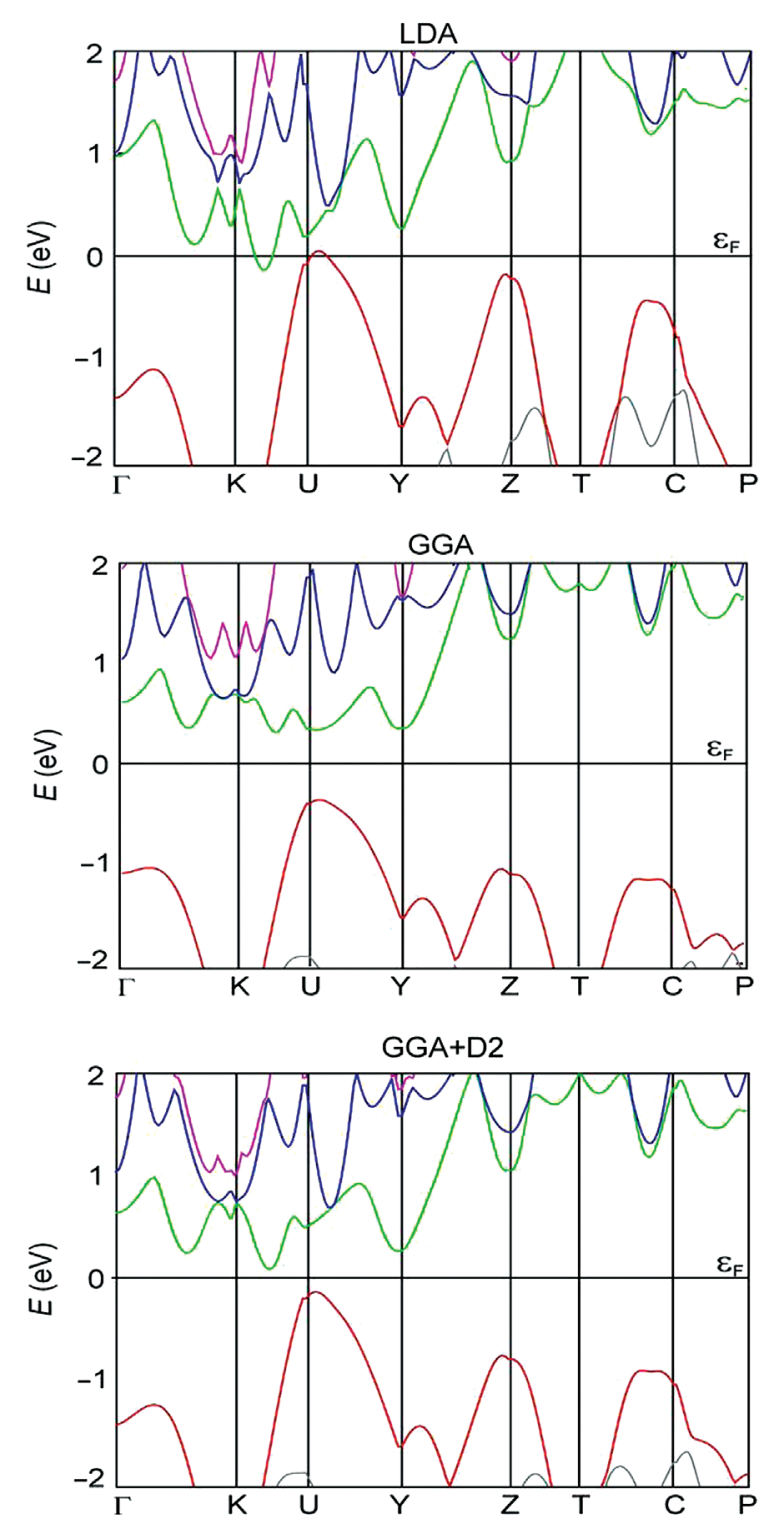

Fig. 6: LDA-, GGA-, and GGA-D2-calculated band structures of orthorhombic black $P$ around the Fermi level. Calculated band gaps are: $0 \mathrm{eV}$ (LDA), $0.70 \mathrm{eV}(\mathrm{GGA})$ and $0.25 \mathrm{eV}$ (GGA-D2). 
electronic structure. In the context of the prediction of an enourmosly dense packing of the phosphorus layers, it incorrectly describes orthorhombic black phosphorus as semimetallic (a closed band gap using the LDA functional is also reported in [50]). While the GGA-calculated band gap of $0.70 \mathrm{eV}$ is too large, the GGA-D2-calculated band gap of $0.25 \mathrm{eV}$ is very close to experimental values of about $0.30-0.35 \mathrm{eV}[67,68]$ for black phosphorus. It has to be emphasized that the effect of the D2 corrections on the calculated band structure are indirect, and entirely due to structural changes.

Finally, the energy values of all allotropes besides white phosphorus are shifted to a narrow range (about $4 \mathrm{~kJ} / \mathrm{mol}$ ) when the dispersion correction is applied. This seems to be very reasonable keeping the chemistry of phosphorus in mind. The occurrence of several allotropes and the comparatively moderate but accurate reaction conditions to achieve these allotropes indicates the presence of similar stabilities. Just little temperature variations can cause a reversion of the relative stabilities. The existence of several metastable allotropes at ambient conditions is subject to kinetic reaction control and very short transition times. White phosphorus represents a special case in this row as it is directly accessible and also the least stable allotrope. Because of the narrow and sensitively temperature-dependent energy region in which the phosphorus allotropes (except white phosphorus) are situated, it is very difficult to make general statements about the order of stabilities. Nevertheless, (only) dispersion corrected calculations confirm black phosphorus as the most stable allotrope, and the relative order of the optimized energy values appears to be reasonable.

\section{Conclusion}

Selected allotropes of phosphorus were investigated within the framework of DFT applying the functionals LDA and GGA. To account for all kinds of interactions including van der Waals forces, the performance of dispersion corrections in combination with the GGA/PBE functional, as suggested by Grimme (GGA-D2), was additionally examined. From LDA and GGA to GGA-D2, the methods as applied show an increasing accuracy due to the types of interactions they cover. For LDA and GGA calculations, the dimension and the relative order of the calculated energy values do not agree well with experimental data. As the simplest functional, LDA fails in the correct description of the energetic order of orthorhombic and trigonal black $\mathrm{P}$ - which is correctly described within GGA. Nonetheless, GGA predicts the tubular allotropes as slightly more stable than black phosphorous - in contrast to experimental data. Additionally, the calculations performed with standard LDA and GGA show strong discrepancies concerning cell geometries. With an increasing role of dispersion interactions in the structures, the calculated cell metrices and sizes differ from the experiment beyond the typical intrinsic deviations. These deviations are also reflected by differences in the electronic structures. By applying the dispersion correction, theoretical and experimental data match much better in terms of both energy values and cell geometries. Taking into account all results, the complete description of the phosphorus system with DFT-D2 provides a benchmark for this method combining accuracy and cost-effectiveness. In this context, it has to be mentioned that the recent developments of D3 [69] and D3BJ [70] came up with a more sound physical background and accuracy [71] to serve as a basis for continuative work on the heavier homologues of phosphorous.

Acknowledgments: The authors would like to thank the Deutsche Forschungsgemeinschaft (DFG, SPP1415, WE 4284/3-2) and the German Academic Exchange Service (DAAD) for financial support.

\section{References}

[1] F. Bachhuber, J. Rothballer, T. Soehnel, R. Weihrich, J. Chem. Phys. 2013, 139, 214705.

[2] F. Bachhuber, J. Rothballer, T. Sohnel, R. Weihrich, Comp. Mater. Sci. 2014, 89, 114.

[3] O. Osters, T. Nilges, F. Bachhuber, F. Pielnhofer, R. Weihrich, M. Schöneich, R. Weihrich, Angew. Chem. Int. Edit. 2012, 51, 2994.

[4] F. Bachhuber, J. von Appen, R. Dronskowski, P. Schmidt, T. Nilges, A. Pfitzner, R. Weihrich, Angew. Chem. Int. Edit. 2014, 53, 11629.

[5] A. Brown, S. Rundqvist, Acta Crystallogr. 1965, 19, 684.

[6] J. C. Jamieson, Science. 1963, 139, 1291.

[7] A. Simon, H. Borrmann, H. Craubner, Phosphorus Sulfur. 1987, 30, 507.

[8] A. Simon, H. Borrmann, J. Horakh, Chem. Ber-Recl. 1997, 130, 1235.

[9] H. Okudera, R. E. Dinnebier, A. Simon, Z. Kristallogr. 2005, 220, 259.

[10] M. Ruck, D. Hoppe, B. Wahl, P. Simon, Y. Wang, G. Seifert, Angew. Chem. Int. Ed. 2005, 44, 7616.

[11] H. Thurn, H. Krebs, Acta Crystallogr. B. 1969, B 25, 125.

[12] M. H. Möller, W. Jeitschko, J. Solid State Chem. 1986, 65, 178.

[13] A. Pfitzner, E. Freudenthaler, Angew. Chem. Int. Ed. 1995, 34, 1647.

[14] A. Pfitzner, E. Freudenthaler, Z. Naturforsch. B. 1997, 52, 199.

[15] A. Pfitzner, M. F. Bräu, J. Zweck, G. Brunklaus, H. Eckert, Angew. Chem. Int. Ed. 2004, 43, 4228.

[16] A. Pfitzner, Angew. Chem. Int. Ed. 2006, 45, 699; Angew. Chem. 2006 118, 714. 
[17] F. Wang, W. K. H. Ng, J. C. Yu, H. J. Zhu, C. H. Li, L. Zhang, Z. F. Liu, Q. Li, Appl Catal B-Environ. 2012, 111, 409.

[18] M. Engel, M. Steiner, P. Avouris, arXivorg, e-Print Arch, Condens Matter. 2014, 1.

[19] H. Liu, A. T. Neal, Z. Zhu, Z. Luo, X. Xu, D. Tomanek, P. Ye, ACS Nano. 2014, 8, 4033.

[20] L. Li, Y. Yu, G. J. Ye, Q. Ge, X. Ou, H. Wu, D. Feng, X. Chen, Y. Zhang, Nat Nanotechnol. 2014, 9, 372.

[21] S. Lange, P. Schmidt, T. Nilges, Inorg. Chem. 2007, 46, 4028.

[22] N. Eckstein, A. Hohmann, R. Weihrich, T. Nilges, P. Schmidt, Z. Anorg. Allg. Chem. 2013, 639, 2741.

[23] S. Böcker, M. Häser, Z. Anorg. Allg. Chem. 1995, 621, 258.

[24] I. Cabria, J. W. Mintmire, Europhys. Lett. 2004, 65, 82.

[25] P. Nava, R. Ahlrichs, Chem. - Eur. J. 2008, 14, 4039.

[26] R. Ahuja, Phys. Status Solidi B. 2003, 235, 282.

[27] A. J. Karttunen, M. Linnolahti, T. A. Pakkanen, Chem. - Eur. J. 2007, 13, 5232.

[28] S. E. Boulfelfel, G. Seifert, Y. Grin, S. Leoni, Phys. Rev. B. 2012, 85, 01411.

[29] S. Appalakondaiah, G. Vaitheeswaran, S. Lebegue, N. E. Christensen, A. Svane, Phys. Rev. B. 2012, 86, 035105.

[30] P. Hohenberg, W. Kohn, Phys. Rev. 1964, 136, B864.

[31] W. Kohn, L. J. Sham, Phys. Rev. 1965, 140, A1133.

[32] N. Ooi, A. Rairkar, J. B. Adams, Carbon. 2006, 44, 231.

[33] L. A. Girifalco, M. Hodak, Phys. Rev. B. 2002, 65, 125404.

[34] G. Kern, J. Hafner, Phys. Rev. B. 1998, 58, 13167.

[35] K. R. Kganyago, P. E. Ngoepe, Mol. Simulat. 1999, 22, 39.

[36] H. Rydberg, N. Jacobson, P. Hyldgaard, S. I. Simak, B. I. Lundqvist, D. C. Langreth, Surf. Sci. 2003, 532-535, 606.

[37] M. Dion, H. Rydberg, E. Schröder, D. C. Langreth, B. I. Lundqvist, Phys. Rev. Lett. 2004, 92, 246401.

[38] K. Lee, É. D. Murray, L. Kong, B. I. Lundqvist, D. C. Langreth, Phys. Rev. B. 2010, 82, 081101.

[39] O. A. Vydrov, T. V. Voorhis, J. Chem. Phys. 2010, 133, 244103.

[40] P. Nozières, D. Pines, Phys. Rev. 1958, 111, 442.

[41] D. C. Langreth, J. P. Perdew, Phys. Rev. B. 1977, 15, 2884.

[42] J. Harl, G. Kresse, Phys. Rev. Lett. 2009, 103, 056401.

[43] J. F. Dobson, T. Gould, J. Phys-Condens. Mat. 2012, 24, 073201.

[44] T. Björkman, A. Gulans, A. V. Krasheninnikov, R. M. Nieminen, Phys. Rev. Lett. 2012, 108, 235502.

[45] S. Grimme, J. Comput. Chem. 2006, 27, 1787.
[46] T. Bučko, J. Hafner, S. Lebègue, J. G. Ángyán, J. Phys. Chem. A. 2010, 114, 11814.

[47] J. Panek, A. Jezierska-Mazzarello. In: Diamond and Related Nanostructures (Eds. M.V. Diudea, C.L. Nagy), p.287. Springer, Netherlands 2013.

[48] V. L. Deringer, R. P. Stoffel, R. Dronskowski, Cryst. Growth Des. 2014, 14, 871.

[49] J.-Y. Noh, H. Kim, J. Korean Phys. Soc. 2012, 60, 410.

[50] O. Prytz, E. Flage-Larsen, J. Phys-Condens. Mat. 2010, 22, 015502.

[51] Y. Du, C. Ouyang, S. Shi, M. Lei, J. Appl. Phys. 2010, 107, 093718.

[52] M. Cascella, I. C. Lin, I. Tavernelli, U. Rothlisberger, J. Chem. Theory Comput. 2009, 5, 2930.

[53] J. P. Perdew, K. Burke, M. Ernzerhof, Phys. Rev. Lett. 1996, 77, 3865.

[54] J. P. Perdew, K. Burke, M. Ernzerhof, Phys. Rev. Lett. 1997, 78, 1396.

[55] J. P. Perdew, Y. Wang, Phys. Rev. B. 1992, 45, 13244.

[56] J. P. Perdew, A. Zunger, Phys. Rev. B. 1981, 23, 5048.

[57] S. Grimme, WIREs Comput. Mol. Sci. 2011, 1, 211.

[58] G. Kresse, J. Non-Cryst. Solids. 1995, 193, 222.

[59] G. Kresse, J. Hafner, Phys. Rev. B. 1994, 49, 14251.

[60] G. Kresse, J. Furthmüller, Comp. Mater. Sci. 1996, 6, 15.

[61] G. Kresse, J. Furthmüller, Phys. Rev. B. 1996, 54, 11169.

[62] P. E. Blöchl, Phys. Rev. B. 1994, 50, 17953.

[63] G. Kresse, D. Joubert, Phys. Rev. B. 1999, 59, 1758.

[64] P. A. G. Ohare, B. M. Lewis, I. Shirotani, Thermochim. Acta. 1988, $129,57$.

[65] R. Weihrich, V. Eyert, S. F. Matar, Chem. Phys. Lett. 2003, 373, 636.

[66] A. Janotti, S. H. Wei, D. J. Singh, Phys. Rev. B. 2001, 64, 174107.

[67] R. W. Keyes, Phys. Rev. 1953, 92, 580.

[68] A. Morita, Appl. Phys. A-Mater. 1986, 39, 227.

[69] S. Grimme, J. Antony, S. Ehrlich, H. Krieg, J. Chem. Phys. 2010, 132, 154104.

[70] S. Grimme, S. Ehrlich, L. Goerigk, J. Comput. Chem. 2011, 32, 1456.

[71] W. Reckien, F. Janetzko, M. Peintinger, T. Bredow, J. Comput. Chem. 2012, 33, 2023. 